\title{
Autologous Porcine Bone Marrow Mesenchymal Cells for Reconstruc- tion of a Resorbed Alveolar Bone: A Preclinical Model in Mini-Pigs
}

\author{
Cecilie Gudveig Gjerde ${ }^{1^{*}}$, Daniele De Santis ${ }^{2}$, Massimo Dominici ${ }^{3}$, Guglielmo Zanotti², Sølve Hellem ${ }^{1}$, \\ Serena Piccinno 3 , Jorge S Burns ${ }^{3}$, Alba Murgia ${ }^{3}$, Olivia Candini ${ }^{3}$, Mauro Krampera ${ }^{4}$, Pierfrancesco \\ Nocini $^{2}$, Alessandro Addis ${ }^{5}$, Jérôme Amiaud ${ }^{6}$, Pierre Layrolle ${ }^{6}$, Kamal Mustafa $^{1}$ and Elena Veronesi ${ }^{3}$
}

\begin{abstract}
${ }^{1}$ Tissue Engineering Group, Institute of Clinical Dentistry, Faculty of Dentistry and Medicine, University of Bergen, Norway

${ }^{2}$ Department of Surgical Sciences, Dental and Maxillofacial Department, University of Verona, Italy ${ }^{3}$ Division of Oncology, Laboratory of Cellular Therapy, University of Modena and Reggio Emilia, Italy ${ }^{4}$ Section of Hematology, Stem Cell Research Laboratory and Cell Factory, Department of Medicine, University of Verona, Policlinico G.B. Rossi Hospital, Italy

${ }^{5}$ CRABCC, Biotechnology Research Center for Cardiothoracic Applications, Italy

${ }^{6}$ INSERM UMR 1238, PHYOS, Laboratory of Pathophysiology of Bone Sarcomas and Remodeling of Calcified Tissues, Faculty of Medicine, University of Nantes, France
\end{abstract}

*Corresponding author: Dr. Cecilie Gudveig Gjerde, Tissue Engineering Group, Institute of Clinical Dentistry, Faculty of Dentistry and Medicine, University of Bergen, Aarstadveien 19, 5008 Bergen, Norway, Tel: +475-558-6610, E-mail: cecilie.gjerde@uib.no

\begin{abstract}
Regeneration of atrophied alveolar bone prior to insertion of dental implants is a major challenge for oral and maxillofacial surgery. It has been reported that Bone Marrow (BM) derived Mesenchymal Stromal Cells (MSC) retain therapeutic potential for bone regeneration.

In the present study, a preclinical mini-pig model simulating the clinical setting was established in order to evaluate the efficacy of autologous MSC for mandible regeneration. Under general anaesthesia, BM aspirates were collected from tibia of mini-pigs $(n=5)$ and MSC were isolated, characterized and expanded. At the same time, a narrow alveolar ridge was simultaneously created by bilateral extraction of two premolar teeth and removal of the buccal bone in order to simulate the pathological situation in humans. After ex vivo expansion, cells were delivered fresh to the surgical operating room and seeded on Biphasic Calcium Phosphate (BCP) granules for 1 hour followed by implantation into the simulated alveolar defects in one pig. The surgical defects were closed with sutures and left to heal for eight weeks. A bone biopsy was taken and dental implants were placed in the newly formed bone.
\end{abstract}

The bone biopsy taken during the procedure showed mineralized bone containing substantial amount of new bone with BCP granules embedded in osteoid tissues and dispersed throughout the newly formed bone matrix. The data demonstrate the osteogenic potential of autologous MSC combined with $\mathrm{BCP}$, providing crucial pre-clinical information in a large animal aimed at the reconstruction of resorbed alveolar bone.

\section{Keywords}

Animal model, MSC, Biphasic calcium phosphate, Mandible augmentation, Histological analysis, Bone regeneration
Abbreviations
BM: Bone Marrow; MSC: Mesenchymal Stromal Cells; BCP: Biphasic Calcium Phosphate; HA: Hydroxy Apatite; $\beta$-TCP: Beta-Tri Calcium Phosphate; PTFE: Polytetrafluoroethylene; CRABCC: Centro di Ricerche ed Applicazioni Biotecnologiche in Chirurgia Cardiovascolare; pMSC: porcine MSC; PBS: Phos- phate Buffered Saline; CFU-F: Colony Forming Unit-Fibro- blasts; PDL: Population Doubling Levels; PMMA: Poly-Methyl Methacrylate; BSEM: Back Scattered Electron Mode 


\section{Introduction}

Extensive bone loss due to trauma, inflammation or ablative tumour surgery may result in large bone defects [1], and reconstruction of these defects remains a major clinical challenge. In the mandible, the volume of the alveolar ridge may also be reduced to a varying degree as a result of congenital malformations, loss of teeth or aging.

Replacement of missing teeth with dental implants is a well-established clinical procedure, but requires an alveolar ridge with a minimum bone width of $6 \mathrm{~mm}[2,3]$. If the width is inadequate, augmentation of the existing bone volume is required [3].

A common approach today to regenerate an alveolar bone involves the application of a barrier membrane to establish a suitable environment for ontogenesis, by excluding connective tissue cells from the bone defect. The barrier membrane maintains locally applied cells close to the bone defect, favouring contribution(s) from adjacent bone osteoprogenitor cells to improve bone healing [4]. Other current therapeutic approaches include the application of bone grafts (autologous, allogeneic, xenogenic), as well as a technique known as segmental bone transport. In particular, autologous bone grafts have become the "gold standard" for bone augmentation since these grafts are osteoconductive, osteoinductive and contain osteogenic cells $[2,5]$. However, there are drawbacks to this approaches it often requires a period of anaesthesia and high number of personnel, resulting in increased costs for the surgical procedures. Additionally, the quantity of the graft is often inadequate. Donor site morbidity is also a concern, with potentially serious sequela such as infections and nerve damage. The risks of bone graft failure, usually attributable to incomplete integration or infection, increases with defect size $[6,7]$.

The search for alternative treatments of bone defects has led to the introduction of Tissue Engineering (TE) strategy combining biomaterials and Mesenchymal Stromal Cells (MSC), as demonstrated in preclinical and early clinical studies [8]. Depending on the clinical indications, different biomaterials may be used as scaffolds for MSC in bone tissue engineering. Moreover, synthetic bone substitutes, such as Hydroxy Apatite (HA) and beta-Tricalcium Phosphate ( $\beta$-TCP) are safe, bioactive and osteoconductive [9]. Several studies have demonstrated the bioactivity of ceramic materials combining $\mathrm{HA}$ and Biphasic Calcium Phosphate (BCP) in varying proportions $[1,10,11]$. Furthermore, the feasibility and efficacy of combining BCP materials and MSC for bone regeneration have been demonstrated in experimental animal models [11-13]. However, for a solid translation of bone tissue engineering from bench to bedside, preclinical testing in large animals is mandatory to ensure suitability and safety for patients. Porcine models are widely applied in regenerative medicine studies, using cells harvested from numerous sites, including bone marrow and adipose tissue. Since mini-pigs have genetic characteristics, gross anatomy, physiology, bone healing patterns and chewing apparatus comparable to those of humans [14], they are increasingly being used to study oral, maxillary and dental procedures $[15,16]$. We therefore designed a study in mini-pigs to simulate the clinical conditions for the reconstruction of resorbed alveolar bone by using autologous culture expanded MSC associated to BCP granules and covered with a titanium reinforced Polytetrafluoroethylene (PTFE) barrier membrane. This study aimed at optimizing protocols for ex-vivo autologous MSC handling, testing surgical procedures and evaluating efficacy of this bone tissue engineering approach in a large animal model using critical sized defects in the alveolar bone.

\section{Material and Methods}

\section{Bone marrow harvest}

The animal experiment was performed according to the Directive 86/609/EEC on the protection of animals used for scientific purposes and conducted at the Centro di Ricerche ed Applicazioni Biotecnologiche in Chirurgia Cardiovascolare, (CRABCC; Rivolta D`Adda, Italy) under the supervision of experienced veterinarians. The preclinical trial was approved by the national competent authority in April 2012 under the Italian past legislative decree D.Lgs 116/92 under the Directive 2010/63/EU. The experimental animals were adult female Yucatan mini-pigs ( $n=6), 16-18$ months old with average weight 60 kilograms. For general anaesthesia, a combination of ketamine and midazolam was used intramuscularly, at doses of $10 \mathrm{mg} / \mathrm{kg}$ and $1 \mathrm{mg} / \mathrm{kg}$ respectively, followed by administration of a mixture of oxygen and isoflurane via facemask and orotracheal intubation. Under general anaesthesia, the tibia was disinfected and using sterile technique $4 \mathrm{ml}$ of Bone Marrow (BM) was aspirated by puncture of the proximal tibia with a 19 or 16 gauge syringe (Matek, Georgia, USA). To help prevent coagulation, heparin (Sigma-Aldrich, St. Louis, USA) $(500 \mu \mathrm{L})$ was added to a final concentration of $130 \mathrm{IU} / \mathrm{mL}$.

\section{Porcine MSC culture and expansion}

BM samples from the mini-pigs were gently agitated during transportation at $4{ }^{\circ} \mathrm{C}$ to the cell culture laboratory. As an initial step towards porcine MSC (pMSC) isolation, an equal volume of Phosphate Buffered Saline (PBS) (Merck-Millipore, Germany) was added to the BM sample (1:1) followed by mixing twenty times by aspiration through a $19 \mathrm{G}$ needle. Red blood cells were then lysed for 5 minutes with gentle agitation at $37^{\circ} \mathrm{C}$ using a $2: 1 \mathrm{v} / \mathrm{v}$ ratio of sterile filtered lysis buffer consisting of $8.56 \mathrm{~g}$ ammonium chloride $\left(\mathrm{NH}_{4} \mathrm{Cl}\right), 1 \mathrm{~g}$ potassium bicar-

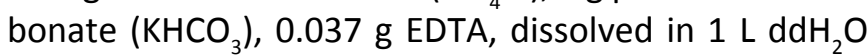
(all from Sigma-Aldrich). The marrow solution was centrifuged at $1200 \mathrm{rpm}$ for ten minutes at $37^{\circ} \mathrm{C}$ and the cells were re-suspended in cell culture medium consist- 


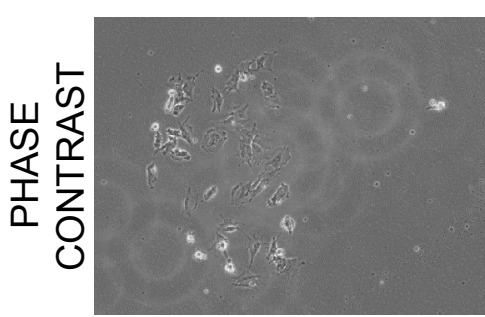

5 days

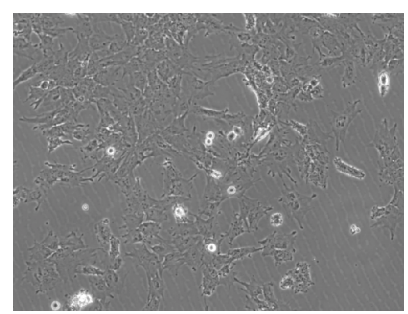

7 days

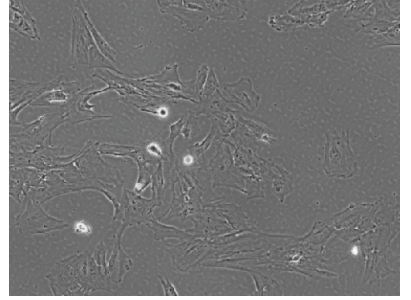

Passage 1

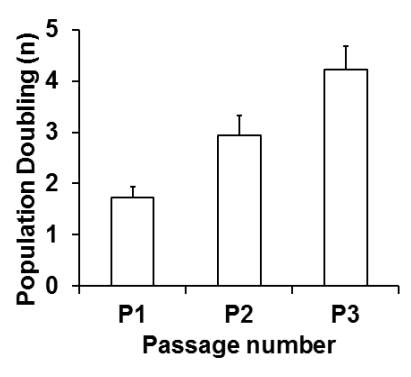

Figure 1: Phase contrast microscopy of pMSC adherent cells 5 or 7 days after seeding and after first passage show consistent cells with a fusiform morphology typical of mesenchymal stromal cells that readily expanded with a relatively constant population doubling rate for the first three passages in monolayer culture.

ing of alpha-MEM $+10 \%$ defined FBS (Stem cell Technologies, Vancouver, Canada). Viable cells were counted using $0.4 \%$ trypan blue dye exclusion. The cells were seeded at $1 \times 10^{6}$ cells $/ \mathrm{cm}^{2}$ in T75 culture flasks (Greiner Bio-One $\mathrm{GmbH}$, Frickenhausen, Germany) and medium was replaced every 4 days of culture in a $37{ }^{\circ} \mathrm{C}, 5 \% \mathrm{CO}_{2}$ humidified incubator (Thermo Scientific, Massachusetts, Stati Uniti). Nine days after seeding, the number of colonies formed was determined by counting Colony Forming Unit-Fibroblasts (CFU-F), quantified relative to the number of cells initially seeded from the isolated BM, according to the formula: (number of colonies/number of cells seeded) $\times 100$. The CFU-F were then detached for cell expansion by incubation with Trypsin $1 X(0.5 \mathrm{mg} /$ $\mathrm{ml}$ ) and EDTA $1 X(0.22 \mathrm{mg} / \mathrm{ml})$ (Euro clone Spa, Milan, Italy) at $37^{\circ} \mathrm{C}$ for 7 minutes; for subsequent passage of confluent flasks the cells were detached using Trypsin 10X (Euroclone) at $37^{\circ} \mathrm{C}$ for 4 minutes, reseeding cells at $10 \times 10^{3} / \mathrm{cm}^{2}$ in $15 \mathrm{~mL}$ culture medium per T75 flask. Cumulative population doublings during the early critical phase of expansion within the first four passages were calculated by counting the viable trypsinized cells using trypan blue dye exclusion, and calculating the number of Population Doubling Levels (PDL) according to the formula: $\mathrm{PD}=\log (\mathrm{N} / \mathrm{NO}) / \log 2$, where NO is the seeded cell number and $\mathrm{N}$ the harvested cell number [17], (Figure 1).

\section{Porcine MSC immuno phenotyping}

Culture expanded pBM-MSC samples were aliquoted in Fluorescence-Activated Cell Sorting (FACS) analysis polypropylene tubes (0.5-1 × 106/tube) (Greiner BioOne $\mathrm{GmbH}$, Frickenhausen, Germany) for immuno phenotype analysis. pBM-MSC were subsequently incubated in the blocking buffer $\left(100 \mu\right.$ leach $0.5-1 \times 10^{6}$ cells $)$ containing DMEM (Gibco, Paisley, UK), 10\% fetal bovine serum (Hyclone, Logan, USA), $0.1 \mathrm{M}$ Sodium Azide and human immunoglobulin G (Sigma-Aldrich, Milan, Italy) and incubated for $20^{\prime}$ on ice. pBM-MSC were washed in PBS (Euroclone, Milan, Italy) and then stained in ice for 30 ' with the follow anti-human monoclonal antibodies: CD45 FITC and CD14 APC (Becton Dickinson, Franklin Lakes, NJ, USA), CD44 FITC and CD31 PE (ebioscience, San Diego, USA), CD29 APC (Biolegend, London, UK), CD90 PE, CD105 FITC and CD73 PE (BD Pharmingen, San
Diego, USA). The pBM-MSC were analysed by FACSARIA equipped with Cell Quest software (Becton Dickinson) and 10,000 events were acquired.

\section{Porcine MSC multipotency}

pBM-MSC were tested for their ability to differentiate into the main mesodermal lineages (adipogenic and osteogenic) as previously described [17]. Briefly, pBM-MSC were induced into adipogenic lineage by culturing in DMEM (Gibco) with addition of $1 \%$ P/S (Gibco, New York, USA), $10 \%$ rabbit serum (Euroclone) and $5 \%$ horse serum (Hyclone) supplemented with dexamethasone $(1 \mu \mathrm{M})$, indomethacin $(60 \mu \mathrm{M})$, Rh-insulin $(10 \mu \mathrm{M})$ and isobutylmethylxanthine $(0.5 \mathrm{mM}$; all from Sigma). The cells were maintained in differentiation media for 10 days and visualization of adipocyte differentiation was performed with of Oil Red O (Sigma-Aldrich, Milan, Italy).

The pMSC osteogenic differentiation potential was measured by treating cell cultures with osteogenic induction medium and subsequently measuring matrix mineralization. Briefly, cells were seeded at $1 \times 10^{4} \mathrm{cell} / \mathrm{cm}^{2}$ per well of a $24-$ multiwell plate. At $60-70 \%$ confluence the cells were treated for 10 days with osteogenic induction medium consisting of expansion medium supplemented with $10 \mathrm{mM} \beta$-glycerophosphate, $0.1 \mathrm{mM}$ ascorbic acid-2-phosphate, $10 \mathrm{nM}$ Dexamethasone (all from Sigma-Aldrich) and $100 \mathrm{ng} / \mathrm{ml}$ recombinant bone morphogenetic protein (rBMP-2, Peprotech, London, UK). Differentiation towards an osteoblastic phenotype was determined based on detection of extracellular matrix mineralization, using a modified Von Kossa method to stain deposits of organic calcium salt [18]. Cultures were fixed in $100 \%$ methanol on ice for 2 minutes and then incubated with $0.1 \%$ silver nitrate in deionized water for 3 hours under a $30 \mathrm{~W}$ UV lamp. A final set of 3 washes of 1 minute each removed non-specific nitrate precipitates. Digitally recorded images of at least ten fields of view per independently isolated MSC population $(n=3)$ were analysed by Image software (National Institutes of Health, USA) to selectively quantify dark positive stained areas of organic calcium deposits.

\section{Shipment of pMSC and their attachment to bioma- terial}

The harvested and expanded pMSC cells were main- 


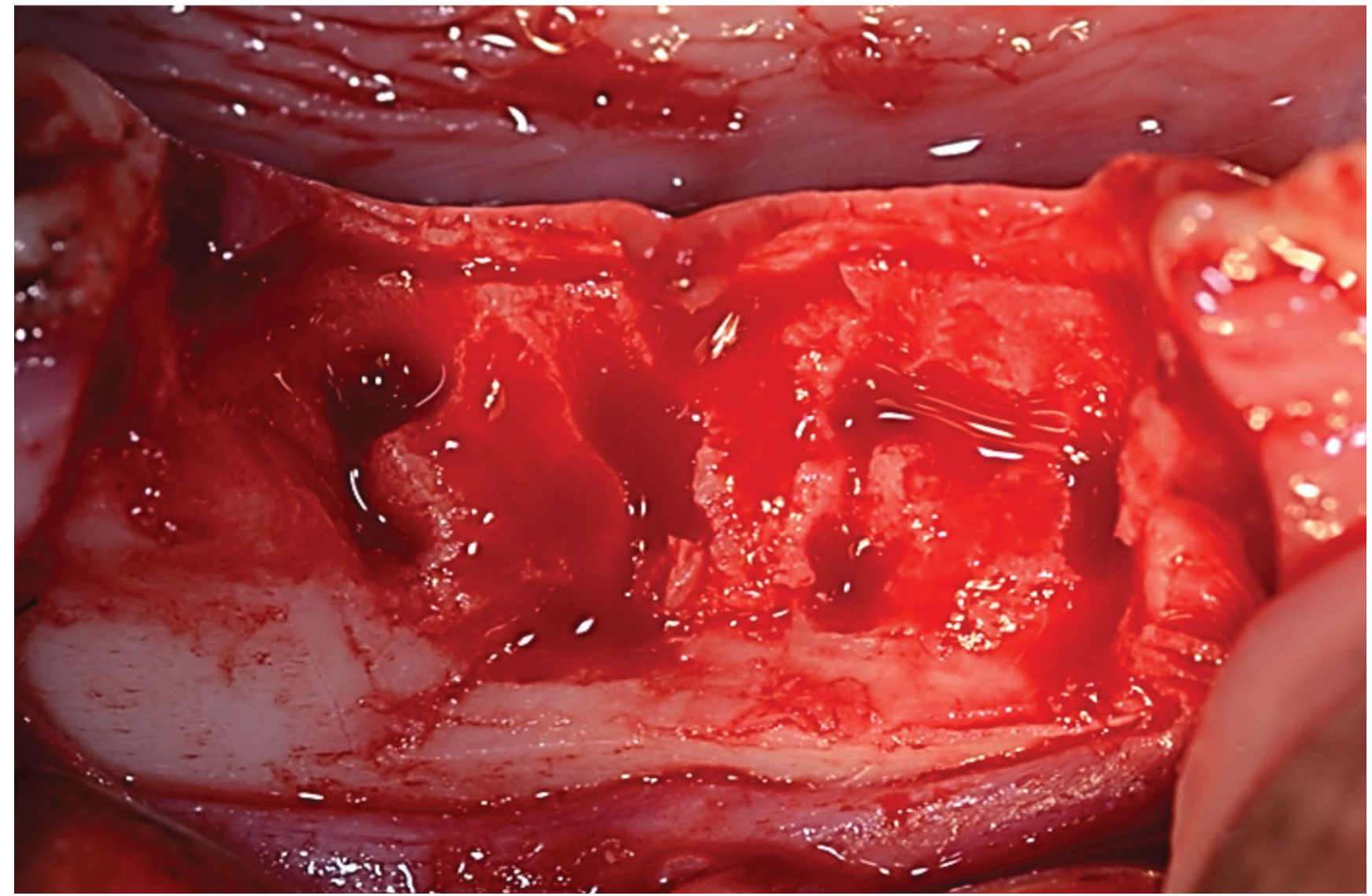

Figure 2: Creation of narrow alveolar bone defects; 2 premolars were extracted and the buccal bone were removed to simulate an strophied alveolar ridge.

tained in $20 \mathrm{ml}$ air-free syringes containing Phosphate Buffered Saline (PBS) at a density of $20 \times 10^{6} \mathrm{cells} / \mathrm{ml}$ at $4{ }^{\circ} \mathrm{C}$ for up to 26 hours to simulate and validate the shipping procedure and condition.

Transportation buffer of $8 \times 10^{5} \mathrm{pMSC} / 100 \mu \mathrm{L}$ were mixed with twenty $1-2 \mathrm{~mm}$ diameter granules $(\approx 20$ $\mathrm{mg}$ ) made of $20 \%$ Hydroxyapatite and $80 \% \beta$-Tricalcium Phosphate (HA/ $\beta$-TCP) (Macro/microporous Biphasic Calcium Phosphate, MBCP+ granules, Biomatlante, France) in a vertically orientated $1 \mathrm{~mL}$ syringes $(n=3)$. The cells were incubated and allowed to attach to the granules for 1 hour at room temperature. The scaffold granules were treated with $0.4 \%$ crystal violet to stain the adherent cells and examined under a microscope. An image-based histological attachment score was used to assess the relative surface area of the scaffold biomaterial covered by pMSC [19].

\section{Surgical procedures}

Under general anaesthesia narrow alveolar ridges were made on the mandibles in one of the animals by bilateral extraction of two premolar teeth and removal of the buccal bone as a split mouth model (Figure 2). The incisions were sutured using monofilament, non-resorbable suture of polypropylene and allowed to heal for two months. During surgery, a solution of the antibiotic enrofloxacin $(5 \mathrm{mg} / \mathrm{ml}$ ) (Bayer HealthCare LLC, Animal Health Division, Pittsburgh, USA) was adminis- tered IM, followed by Amoxicillin long acting $(10 \mathrm{mg} /$ kg), (Clamoxyl L.A. 250 ML Zoetis, Pfizer) IM for 10 days postoperatively. After extubation the pig was returned to the enclosure and confined for the first postoperative week. As a prophylactic analgesic, meloxicam, 0.4 $\mathrm{mg} / \mathrm{Kg}$ (Metacam Injection $20 \mathrm{mg} / \mathrm{ml} 100 \mathrm{ml}$ Boehringer Ingelheim) was administered intramuscularly, followed by IM administration of meloxicam, $0.4 \mathrm{mg} / \mathrm{Kg}$ (Metacam Injection $20 \mathrm{mg} / \mathrm{ml} 100 \mathrm{ml}$, Boehringer Ingelheim) for 7 days after surgery.

After healing for two months, under general anaesthesia the alveolar ridge was inspected and the sites were re-entered from the canine and distally to the first molar (Figure 3). The cortical bone on both sides was punctured and 2 tenting mini screws were inserted. Autologous cultured expanded pMSC were mixed with the MBCP granules and cells allowed to attach to the granules for 1 hour and then grafted into the test site. A dose of $50 \times 10^{6} \mathrm{pMSC}$ suspended in $2.5 \mathrm{ml}$ of physiological saline was mixed with $2.5 \mathrm{~cm}^{3}$ of BCP granules of $1-2 \mathrm{~mm}$ in size packaged in a syringe using a double Luer lock. Biomaterial without MSC was placed on the contra lateral site in this split mouth model and used as a control site. Dense PTFE titanium reinforced membranes were stabilized over the grafts (Figure 4) by pins to the lingual and buccal sides (Cytoplast ${ }^{\circledR}$ PTFE titanium reinforced membrane and Profix ${ }^{\circledR}$ pins and screws, OSTEOGENIC BIOMEDICAL $^{\circledR}$, USA). For total closure of the wound, the 


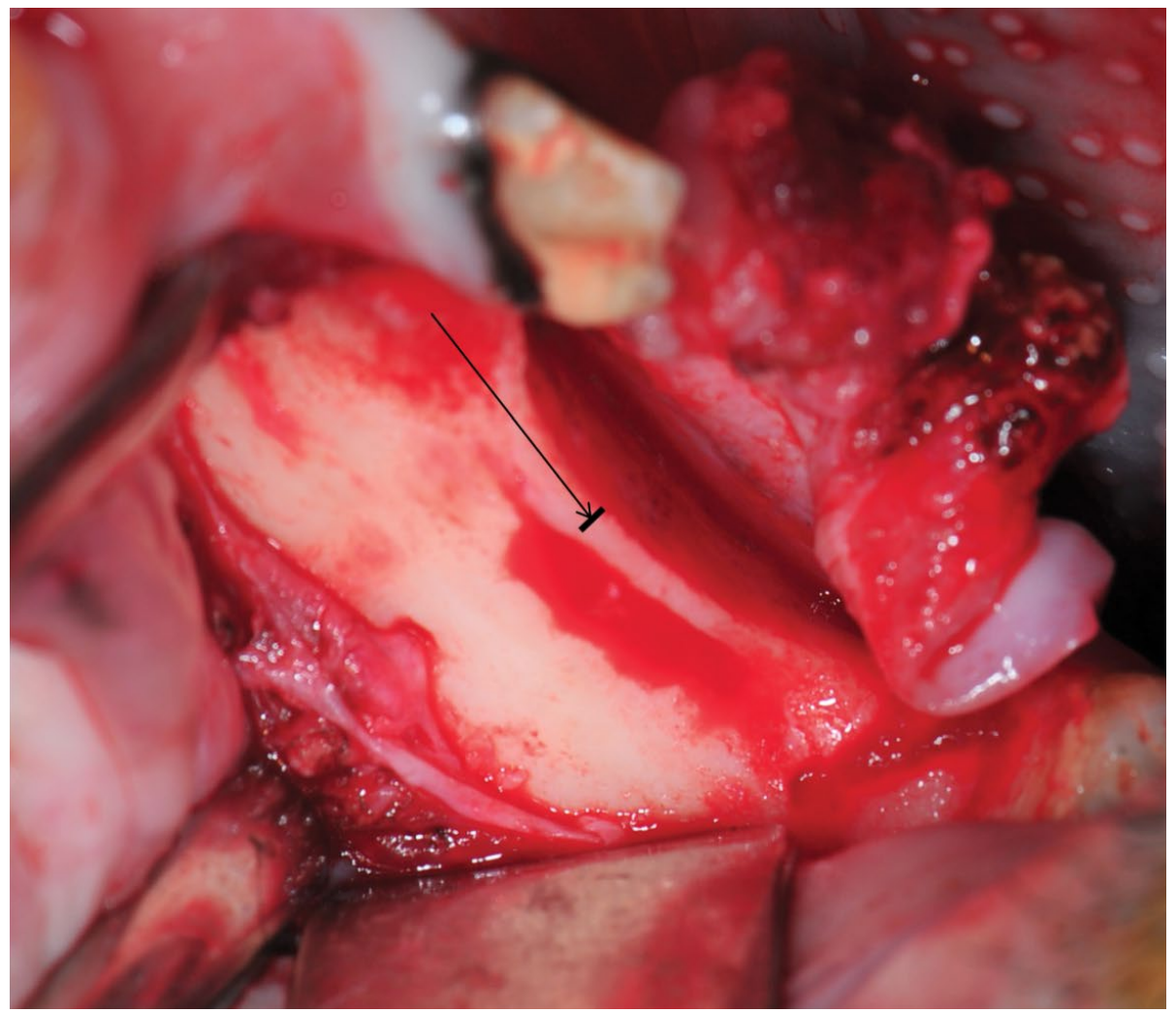

Figure 3: Mandibular alveolar ridge, 2 months of healing after creation of the narrow bone defects and before augmentation. Arrow pointing at the line indicating the width of the ridge.

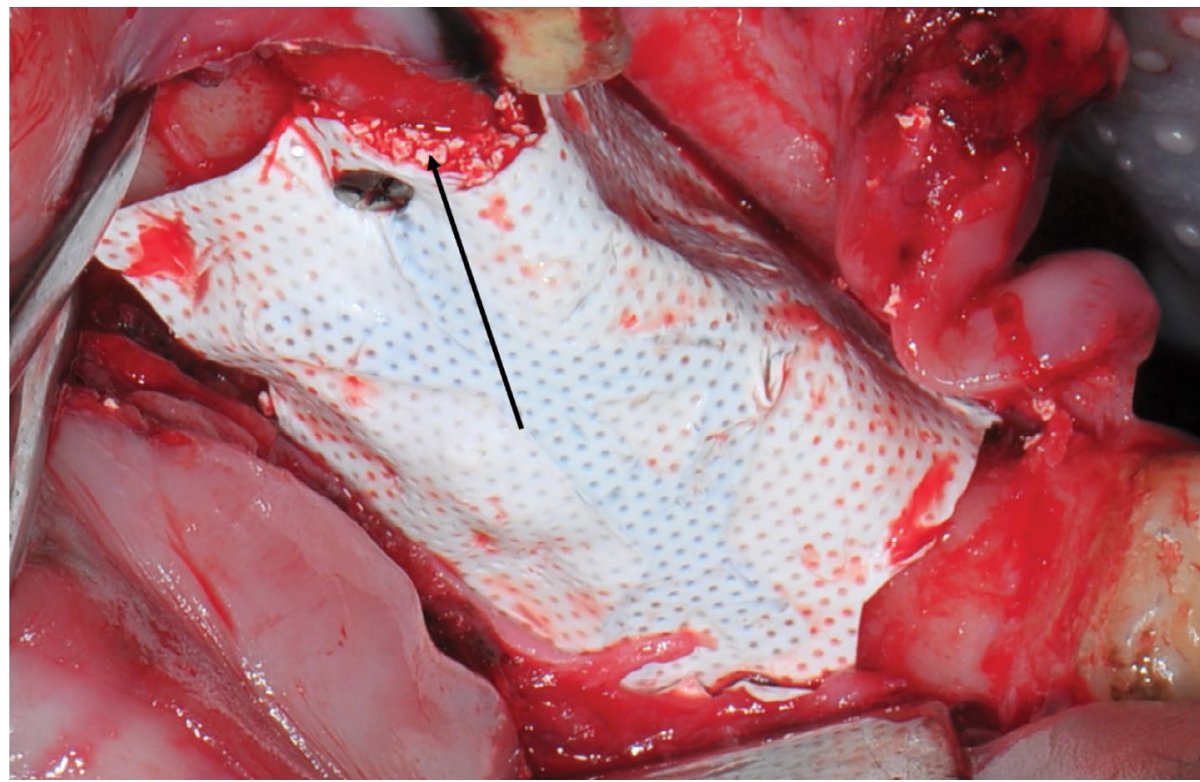

Figure 4: The mixture of stromal cells and $\mathrm{MBCP}+$ in place under the membrane. Arrow points to the mixture of granules and pMSC.

mucoperiosteum was stabilised with mattress and single sutures. During surgery and postoperatively, antibiotics and analgesics were administered as described above. After 10 days, the sutures were removed uneventfully, under sedation. The pig was kept on a soft diet for the remaining experimental period.

After further 60 days of healing the pig was deeply anaesthetised and sacrificed. Bone biopsies were taken from test and control sites using a trephine burr (But- terfly ${ }^{\circledR}$, Italy). At the test site, 2 dental implants (Nobel Biocare ${ }^{\circledR}$, Switzerland) were installed and tested for stability (Osstell ${ }^{\circledR}$ ISQ; Osstell, Goteborg, Sweden).

\section{Histology}

After fixation in neutral formaldehyde $10 \%$, the biopsies were processed for non-decalcified histology using Polymethyl Methacrylate (PMMA) resin. The biopsies were then dehydrated by using ascending concentrations of eth- 
anol baths at $4{ }^{\circ} \mathrm{C}(70 \%, 80 \%, 95 \%$ and $100 \%$ ethanol) and finally by soaking for 48 hours in acetone. The biopsies were then impregnated with a mixture of 90/10 vol. of methyl methacrylate (Sigma-Aldrich) and Poly-Ethylene Glycol 400 (PEG, Fluka) for 48 hours at $-20^{\circ} \mathrm{C}$ under vacuum in order to avoid bubbles during polymerization. Finally, the biopsies were embedded in PMMA at $-20^{\circ} \mathrm{C}$ under vacuum using an initiator ( $0.8 \mathrm{wt}$. \% benzoyl peroxide, Merck) and propagator $(60 \mu \mathrm{l} / 5 \mathrm{ml}$ of $1 \mathrm{ml} \mathrm{N}$, N-dimethyl aniline/19 $\mathrm{ml}$ propanol-2) of radical polymerization. The blocks were cut medially in two parts: One part was used to perform a descriptive analysis of the augmented volume, while the second part served for Scanning Electron Microscopy (SEM, Hitachi, TM3000, Japan) and subsequent quantitative analyses of newly formed bone and the remaining biomaterial. A circular inner lock diamond saw (LeicaSP1600, Germany) and a grinder/polisher (Bluewher Metaserv 250, Germany) were used to prepare $30 \mu \mathrm{m}$ thick sections mounted on polycarbonate slides using epoxy glue. The sections were stained using methylene blue (1\%) for $90 \mathrm{~s}$ and basic fuchsin $(0.3 \%)$ for 25 seconds. This staining revealed mineralized bone tissue in purple pink while non-calcified soft tissues remain blue purple, and nuclei are stained blue. Histology sections were finally scanned (NanoZoomer 2.0RS, Hamamatsu Corp. Japan) and observed using a virtual micro- scope (NDP view software, Hamamatsu Corp. Japan). For SEM observations, blocks were polished using Si C paper and cleaned. Contiguous photographs of the blocks were made at magnification X50 by using the Back Scattered Electron Mode (BSEM), Hitachi, Tabletop TM3000). On these SEM images, calcium phosphate granules appeared grey/white, mineralized bone in grey and fibrous tissue in black.

\section{Statistics}

A two-tail Student's t-test (assuming equal variances) was used to determine the statistical significance of the differences in the dark positive stained areas of organic calcium deposits. P-Values $<0.05$ were considered statistically significant.

\section{Results}

\section{Isolation, culture and expansion of autologous $\mathrm{Yu}$ - catan mini-pig MSC ex vivo}

The harvest volume of less than $6 \mathrm{~mL}$ of porcine BM tissue yielded approximately $3.15 \times 10^{5} \pm 9 \times 10^{3}$ cells $/ \mathrm{mL}$ after red blood cell lysis. Of note, in 2 out of 5 harvests, the procedure had to be interrupted due to clotting of the harvested BM cell suspension despite the anti-clotting agent introduced. The issue was solved by repeat-

\section{A}

\begin{tabular}{|c|c|}
\hline MARKER & $\begin{array}{c}\text { Mean } \pm \text { SEM } \\
(\mathbf{n}=\mathbf{3})\end{array}$ \\
\hline CD45 & $4.85 \pm 4.55$ \\
\hline CD14 & $6.35 \pm 6.25$ \\
\hline CD31 & $0.35 \pm 0.25$ \\
\hline CD44 & $99.60 \pm 0.20$ \\
\hline CD90 & $99.35 \pm 0.45$ \\
\hline CD29 & $98.95 \pm 0.05$ \\
\hline CD105 & $4.00 \pm 0.35$ \\
\hline CD73 & $0.55 \pm 0.15$ \\
\hline
\end{tabular}
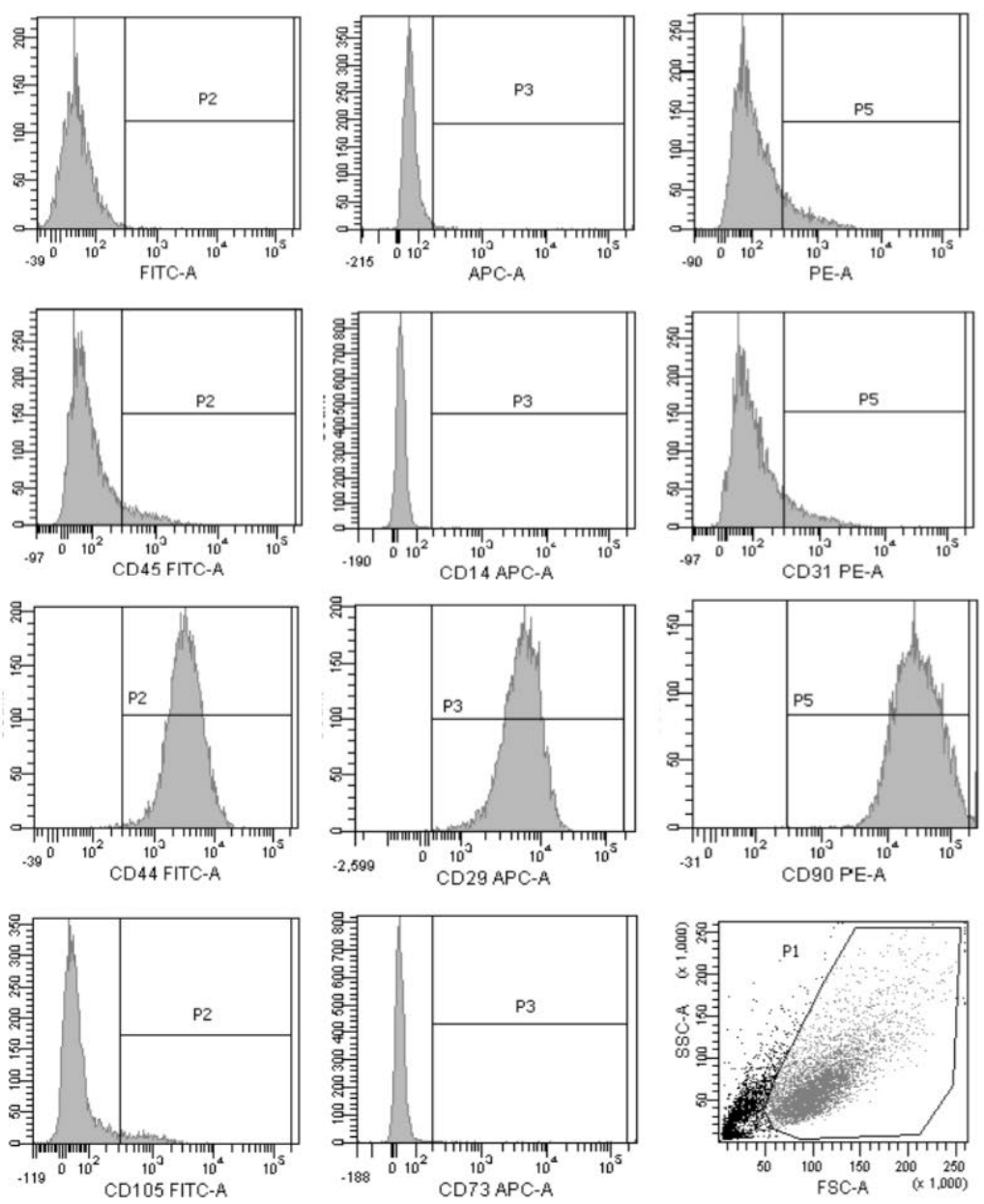

Figure 5: A,B) Flowcytometry analysis of pBM-MSC surface makers expression. The histogram indicates the negative and positive stained cells. 
ing the harvest and maintaining the freshly harvested samples in constant agitation using a Biotech Macs mix Tube Rotator (Miltenyi, Germany) at $+4{ }^{\circ} \mathrm{C}$. After 10 days of culture, there was an average of $6.43 \pm 1.2 \mathrm{CFU}-\mathrm{F} /$ million seeded cells. Cells were spindle-shape adherent cells that readily proliferated in culture, similar to their human counterparts. Once detached cells were proliferating with a cumulative PD of $4.22 \pm 0.45$ at passage 4 .

\section{Porcine MSC characterization and multipotency}

In order to characterize cells isolated from pig bone marrow, we performed immuno phenotype analysis with the follow anti-human monoclonal antibodies: CD45, CD14, CD31, CD44, CD29, CD90, CD105 and CD73. As seen on Figure $5 \mathrm{~A}$ (dot plot on right in upper panel) cells display physical parameter typical of stromal cells population, being negative for CD45, CD14 and CD31 (Figure 5B bottom panel) and positive for CD44 (99.60 $\pm 0.20), \operatorname{CD} 29$ (98.95 \pm 0.05$)$ and CD90 (98.35 \pm 0.45$)$. CD73 and CD105 were not been detected confirming the results previously obtained from Noort, et al. [20] that distinguish these cells from human marrow MSC.

Multi lineage potentials of isolated pMSC was assessed at Passage 4 (P4) inducing differentiation towards osteogenic and adipogenic. The cells were able to generate round lipid droplets appearance inside the cells and Oil Red O staining confirmed the adipose commitment (Figure 6A). The response of pMSC monolayer cultures to osteogenic medium included the formation of dense multicellular foci with an extracellular matrix that was subsequently stained black by the Von Kossa stain for organic calcium salts. Typically, about $6 \%$ of the monolayer surface was strongly Von Kossa positive, significantly more than the control cultures $(p<0.05)$ (Figure 6B).

\section{MSC adhesion to 3D scaffolds after cell transpor- tation}

Using a cell number to scaffold weight ratio consistent with that envisaged for clinical situations, the ability of freshly harvested pMSC to adhere to the 3D HA- $\beta$ TCP scaffolds was significantly greater than that of pMSC after shipping at high cell density in a saline transport buffer at $4{ }^{\circ} \mathrm{C}$. Nonetheless, although reduced, the adhesion potential of pMSC after exposure to shipping conditions was $>70 \%$ of that exhibited by the control cells not exposed to shipping conditions, which would be adequate for graft preparation (Figure 7).

\section{Autologous pMSC with BCP granules generate abundant bone formation in vivo}

In one mini-pig, a critical sized split mouth model was used in a pilot study to investigate the efficacy of autologous pMSC combined with the MBCP+ ceramic material. During the experimental period the animal remained in good health and there were no wound healing complications or any signs of infection. Two months after the mandibular graft, biopsies were taken with trephine burs prior to implant insertion to evaluate new bone formation within the defect sites. As shown in Figure 8 , abundant formation of bone tissue was observed around the $\mathrm{MBCP}+$ granules at the test site.

Further SEM observations corroborated the histological analyses (Figure 9). In biopsies of the control site without cells, a limited quantity of mineralized bone (grey) had formed around the MBCP+ granules (white). In contrast, abundant newly formed bone was observed in biopsies of the test site with pMSC combined with $\mathrm{MBCP}+$ granules.
A

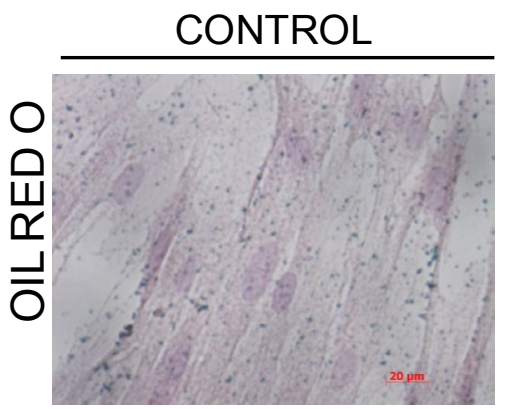

B

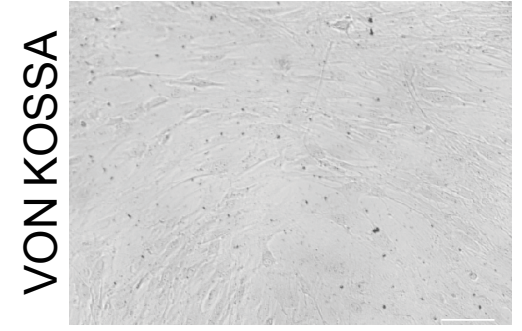

INDUCED
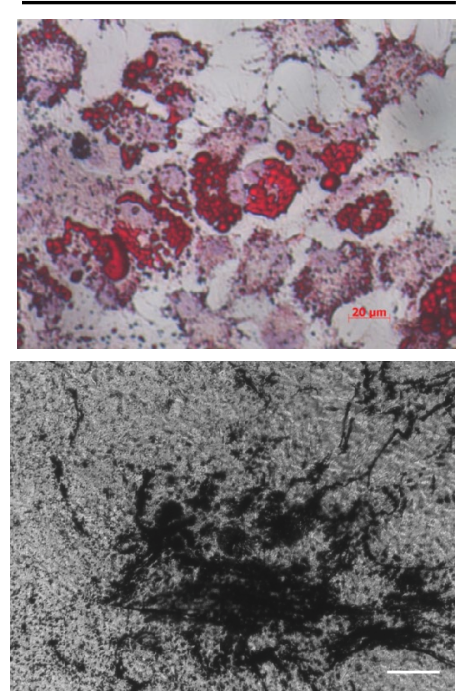

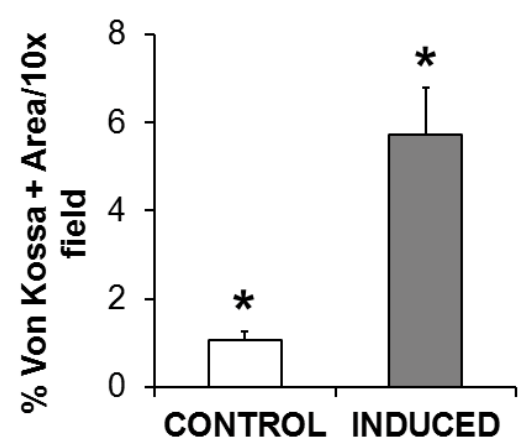

Figure 6: A) Adipogenic differentiation of pBM-MSC by Oil-Red-O (Sigma) after 10 days. Micropictures at magnification 20X were acquired with invert optic Observer (Zeiss, Munic, Germany) equipped with Axiovison 4.2.2 software (Zeiss); B) Photomicrographs of Von Kossa stained cell monolayers showed that in contrast to control pMSC, cells induced by BMP-2 treatment had significantly more black stained areas $\left(>5\right.$-fold, $\left.{ }^{*} p<0.05\right)$ indicative of ex vivo mineralization. 

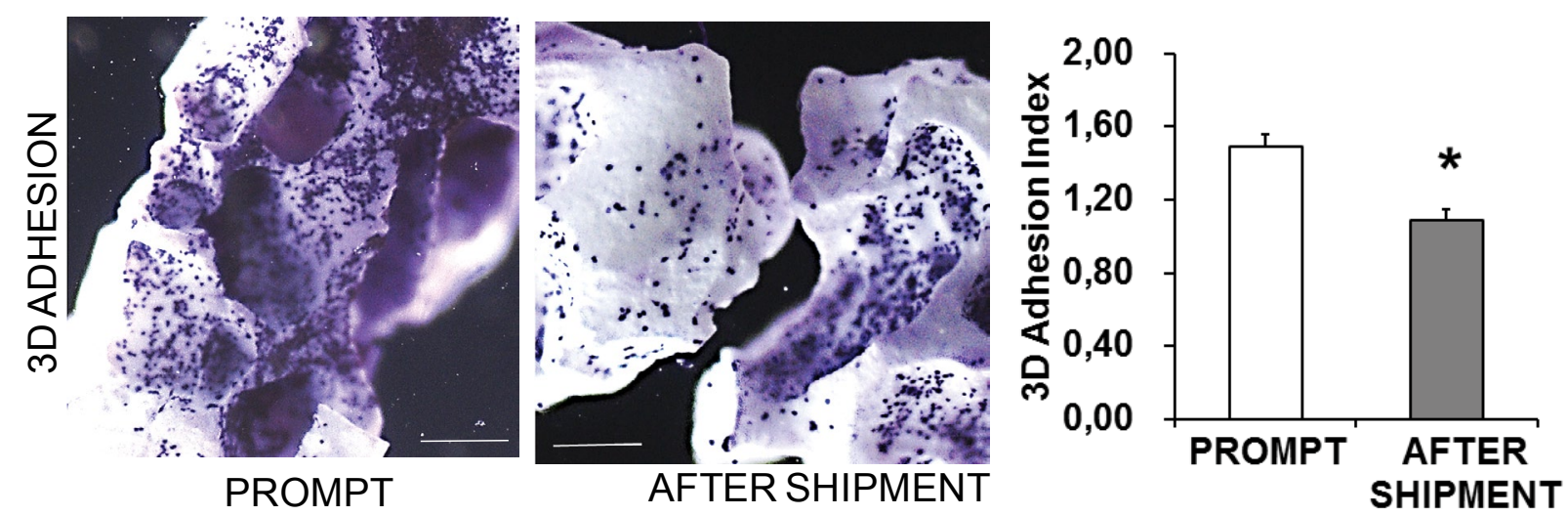

Figure 7: Phase contrast photomicrographs of blue stained cells adhering to the 3D surface of the HA/ $\beta-T C P$ scaffold. In contrast to promptly used control cells, the $3 \mathrm{D}$ attachment-index after shipment was significantly reduced $\left({ }^{*} p<0.05\right)$, but still over $70 \%$ of the control value. Bar $=100 \mu \mathrm{m}$.

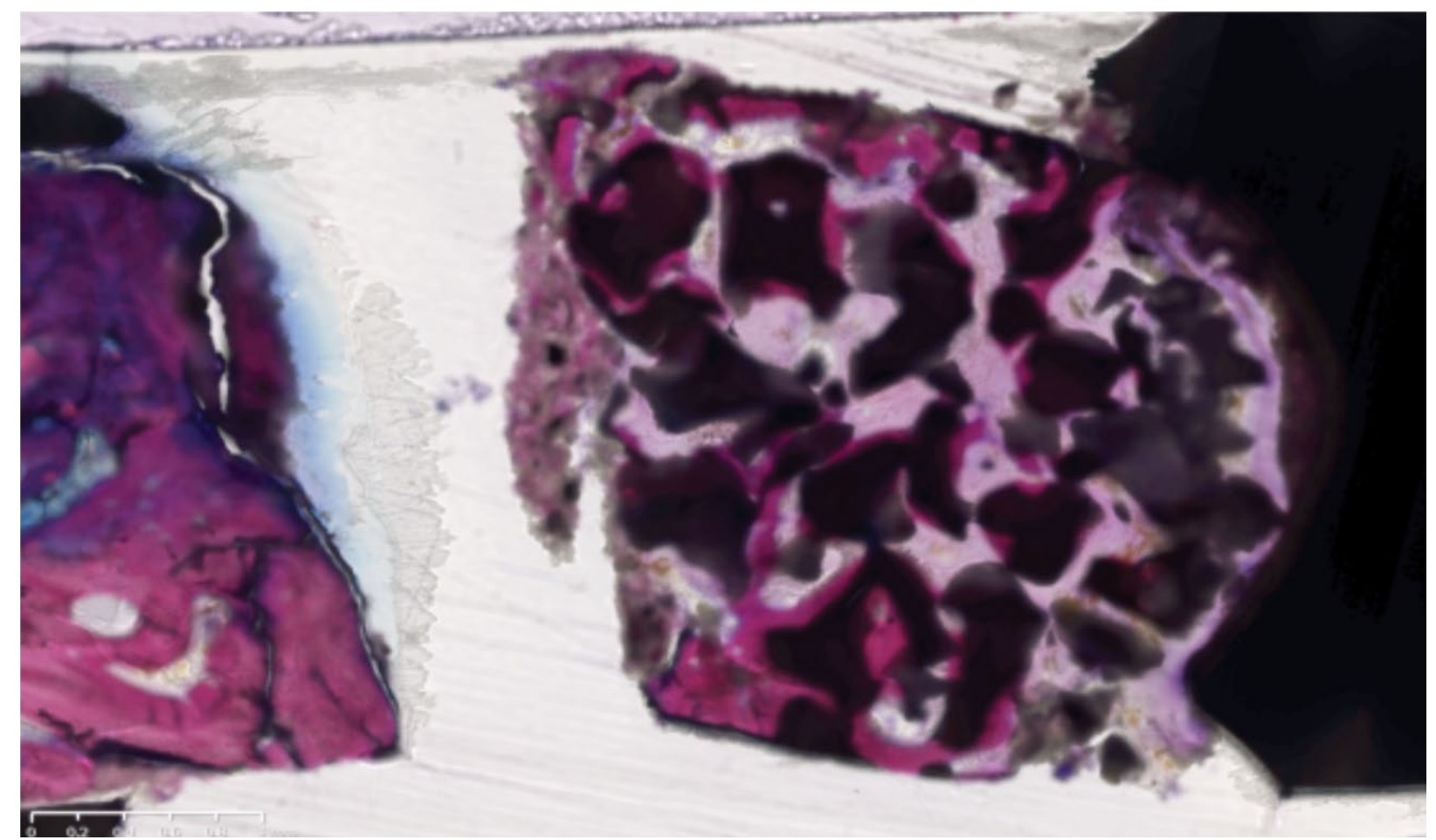

Figure 8: Evaluation of newly formed bone in response to $\mathrm{MSC} /$ biomaterial graft. A representative histological sample stained with methylene blue (1\%) and basic fuchsin $(0.3 \%)$.

The membrane in the test side with the pMSC remained intact under the mucosa (Figure 10). At the control site, the membrane was exposed and no healing had occurred. A substantial amount of bone is seen under the membrane (Figure 11). In this context, new bone had formed in between and in contact with the MBCP+ granules.

\section{Discussion}

The main finding in this study is that the combination of autologous pMSC and biomaterial was used successfully to augment the alveolar ridge in the mini-pig. To the best of the author's knowledge similar results has not been published before. The present study was performed to assess whether procedures for isolation and ex vivo expansion of autologous BM-derived MSC harvested from mini-pigs could be used to develop a large-animal model of a tissue engineering approach to mandible regeneration. This preclinical model confirms the adipogenic and osteogenic potential of pMSC in vitro. Further, it shows that ex vivo expanded pMSC can adequately survive at high cell density under shipment conditions consistent with our previous data in humans [19]. In addition, the results show that after shipment, mini-pig MSC are fully capable of adhesion to the 3D scaffold biomaterial within a one-hour time frame, thereby meeting the requirements for delivery of cells to a surgical team in a surgical operating room.

Based on the literature on reconstruction of alveolar ridges in the atrophied lower jaw, the consensus has been that reconstruction here is more difficult because 

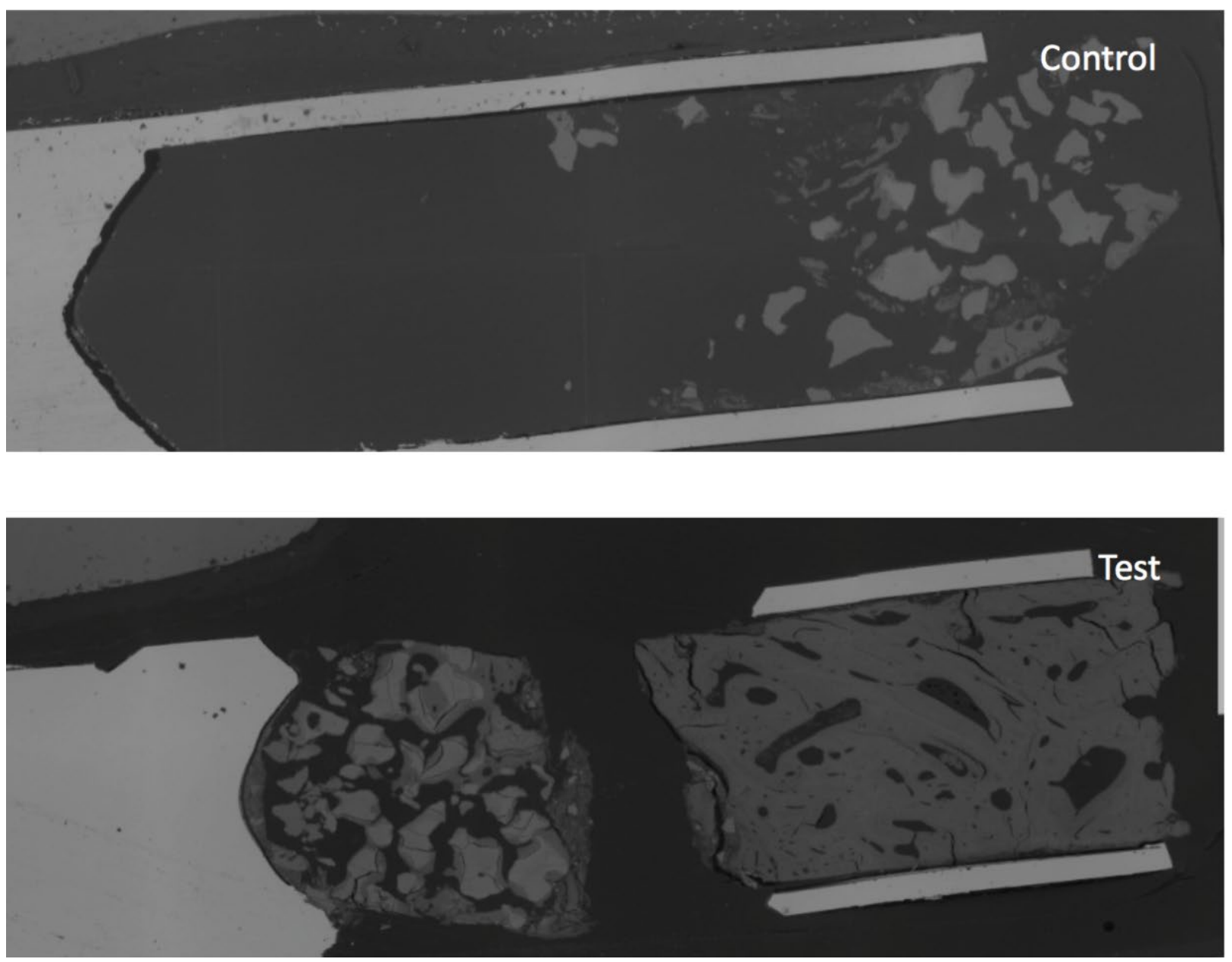

Figure 9: Microgrpahs from a scanning electron microscope, the blocks were made at magnification X50. On these images, calcium phosphate granules appeared grey/white, mineralized bone in grey and fibrous tissue in black.

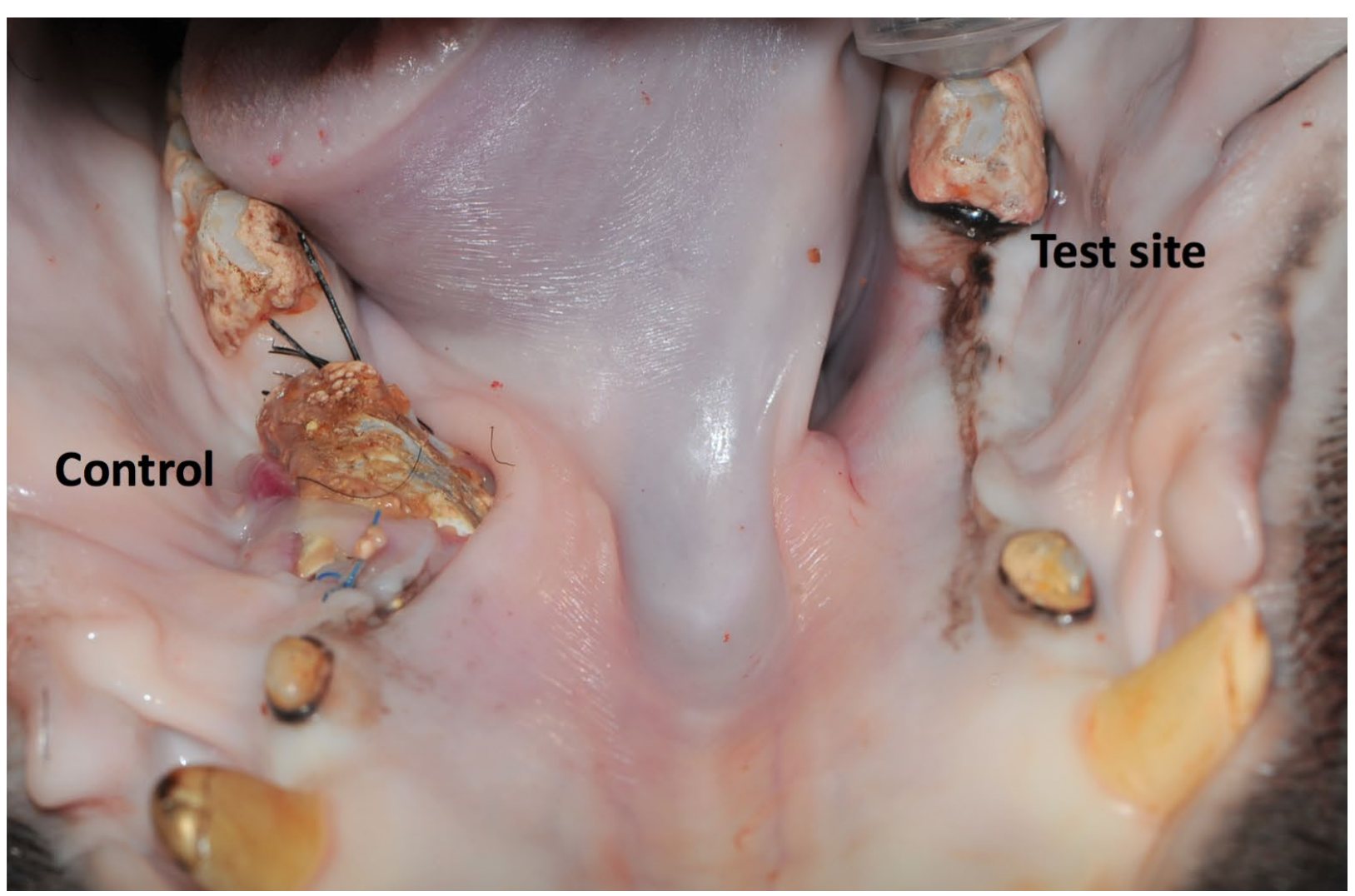

Figure 10: Bone and soft tissue healing after 8 weeks. Control side on left where the membrane is exposed, on the right test side a favorable healing outcome is shown. 


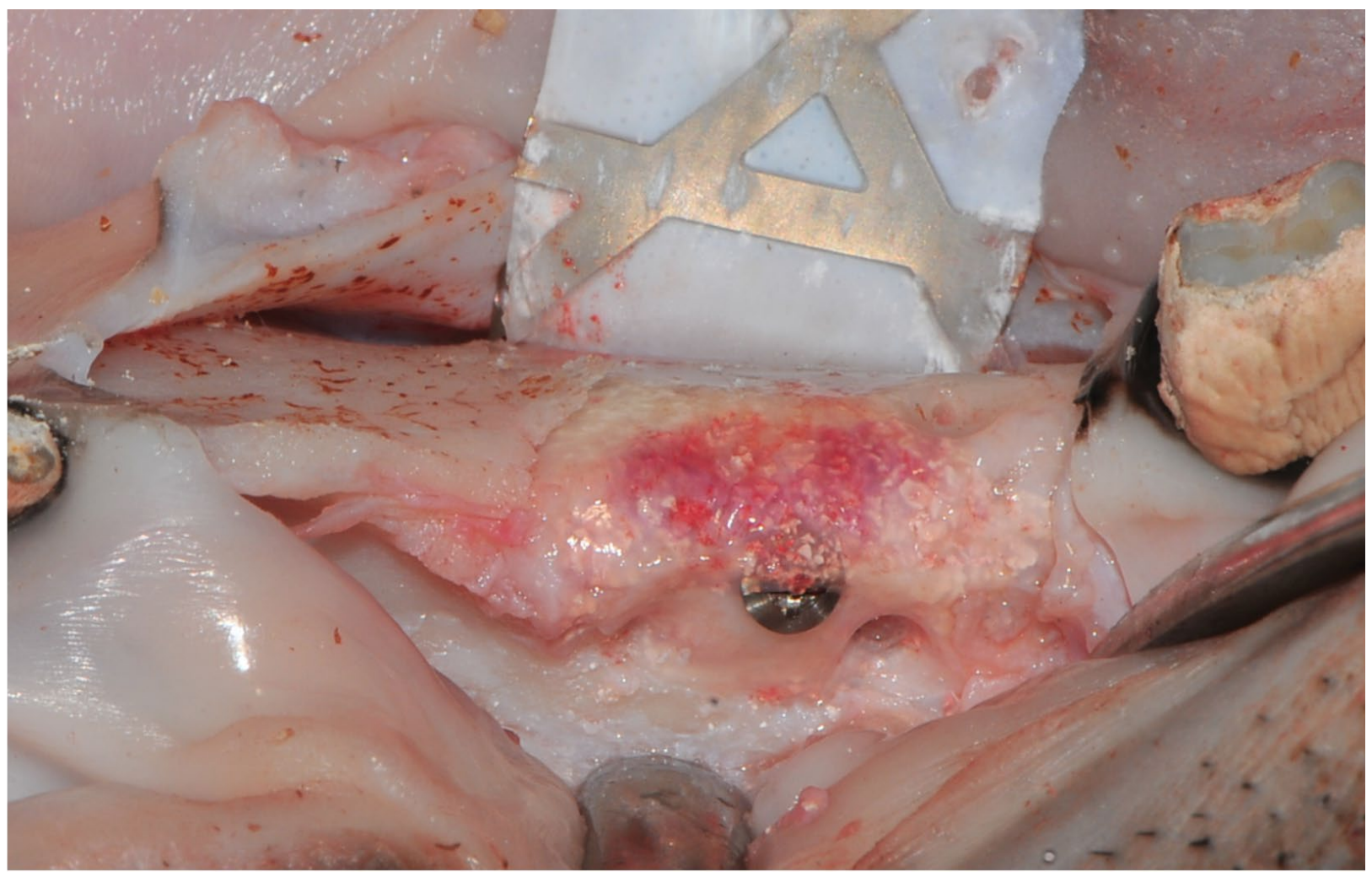

Figure 11: Bone healing on the test site where a combination of MSC and biomaterial were used. The granules are still visible, but the newly formed bone is shown.

of limited blood supply, extensive chewing force and lack of compliance by the pig with respect to soft diet and oral hygiene [21]. In a similar mini-pig trial using a bone graft to reconstruct the mandibular ridge, the authors did not recommend the intraoral approach because of complications in healing of the gingival wound [22]. The posterior mandible in particular is the most difficult area to reconstruct because of limited blood supply and the high proportion of cortical bone $[2,23]$. Further, as the oral cavity harbours a relevant microbiological burden, the risk of post-operative infection is thought to be high. While limited to a single animal, the MSC-augmented area in the mini-pig mandible in the present study showed complete remodelling of the defect, a result that exceeded original expectations. The data also demonstrated that using only BCP granules as bone substitute might not be enough to induce bone formation on severely atrophied mandibular bone.

Because of the physiological similarities, translation of the results from pigs to humans is more relevant than from other experimental animals such as mice and rabbits [14]. Indeed, the pig is an established animal model for dental, oral, and maxillofacial research and preclinical testing, including novel implant design testing [15], since porcine bone anatomy, morphology, healing and remodelling are similar to those of humans. In particular, bone regeneration in humans is $1.0-1.5 \mathrm{~mm} /$ day with the reported rate for pigs (1.2-1.5 mm/day) being closer than that for dogs (1.5-2.0 mm/day) [16].

Harvesting of BM-MSC in the mini-pig has been chal- lenging, also for the high tendency of BM aspirate to clot, despite the anti-clotting agents used. In humans, the iliac crest is a common site for collection of bone marrow. However, the bone structure of swine is massive and the cortical thicknesses are generally much greater than in other species and in humans. Similar problems are encountered with collection of BM from the sternum, except in neonatal swine. Consequently, in mini-pigs the tibia has been considered the easiest site from which to obtain BM samples as a logical extension of current surgical procedures. However, despite the central role of the porcine model in continued medical advances in the field, only limited information is available about porcine bone marrow MSC.

\section{Conclusions}

The results of this study show that pMSC are capable of generating bone, and that this new bone integrated with the surfaces of the recipient bone as well as with the BCP and re-established the volume of the alveolar ridge. However, as these promising results are based on observations from a single animal only, further investigation is warranted.

\section{Funding}

This work was supported by a grant from the European Union's Seventh Framework Programme under grant agreement number 241879 - REBORNE 'Regenerating bone defects using new biomedical engineering approaches'. 


\section{Authors Contributions}

CGG: Designing the protocol of the study, performing the experiments, surgical part, writing manuscript; DDS: Designing protocol, participating in the animal model, writing manuscript; MD: Designing protocol, performing the in vitro experiments and assays, writing manuscript; GZ: Contributing to the surgery, writing manuscript; SH: Designing protocol, writing manuscript; JSB: Contributed to the animal experiment, writing manuscript; EV, SP, $A M$ and OC: Performing the in vitro experiment (MSC expansion ex vivo); $\mathrm{MK}$ and PN: Designing protocol; $\mathrm{AA}$ : Contributed to the animal experiment; JA: Histological analysis; PL: Designed and coordinated the project; KM: Designing the protocol and writing manuscript.

\section{Declarations}

Ethics approval: The animal experiment was performed according to the Directive 86/609/EEC on the protection of animals used for experimental and other scientific purposes and conducted at the Centro di Ricerche ed Applicazioni Biotecnologiche in Chirurgia Cardiovascolare, (CRABCC; RivoltaD`Adda, Italy) under the supervision of experienced veterinarians. The preclinical trial was approved by the national competent authority in April 2012 under the Italian past legislative decree D. Lgs 116/92 under the Directive 2010/63/EU.

\section{Consent for Publication}

Not applicable.

\section{Competing Interest}

The authors declare no competing interests.

\section{Acknowledgements}

The authors are in debtto M Campagnoli at the CRABCC, Italy for his excellent veterinary assistance.

\section{References}

1. Sarkar SK, Lee BT (2015) Hard tissue regeneration using bone substitutes: An update on innovations in materials. Korean J Intern Med 30: 279-293.

2. Chiapasco M, Casentini P, Zaniboni M (2009) Bone augmentation procedures in implant dentistry. Int $\mathrm{J}$ Oral Maxillofac Implants 24: 237-259.

3. Coulthard P, Esposito M, Jokstad A, Worthington HV (2003) Interventions for replacing missing teeth: Bone augmentation techniques for dental implant treatment. Cochrane Database Syst Rev.

4. Schenk RK, Buser D, Hardwick WR, Dahlin C (1994) Healing pattern of bone regeneration in membrane-protected defects: A histologic study in the canine mandible. Int J Oral Maxillofac Implants 9: 13-29.

5. Albrektsson T, Johansson C (2001) Osteoinduction, osteoconduction and osseointegration. Eur Spine J 10: S96-S101.

6. Garg AK (2004) Bone Biology, Harvesting, and Grafting for Dental Implants: Rationale and Clinical Applications. Quintessence Publishing, USA, 279.

7. Marx RE, Morales MJ (1988) Morbidity from bone harvest in major jaw reconstruction: A randomized trial comparing the lateral anterior and posterior approaches to the ilium. J Oral Maxillofac Surg 46: 196-203.

8. Shanbhag S, Shanbhag V (2015) Clinical applications of cell-based approaches in alveolar bone augmentation: A systematic review. Clin Implant Dent Relat Res 17: e17-e34.

9. Kneser U, Schaefer DJ, Polykandriotis E, Horch RE (2006) Tissue engineering of bone: The reconstructive surgeon's point of view. J Cell Mol Med 10: 7-19.

10. Hornez JC, Chai F, Monchau F, Blanchemain N, Descamps $\mathrm{M}$, et al. (2007) Biological and physico-chemical assessment of Hydroxyapatite (HA) with different porosity. Biomol Eng 24: 505-509.

11. Veron C, Chanavaz M, Ferri J, Donazzan M, Hildebrand HF (1995) A panorama of current materials for osseous application in maxillofacial surgery and oral implantology. Rev Stomatol Chir Maxillofac 96: 274-281.

12. Arinzeh TL, Tran T, McAlary J, Daculsi G (2005) A comparative study of biphasic calcium phosphate ceramics for human mesenchymal stem-cell-induced bone formation. Biomaterials 26: 3631-3638.

13. Gomez-Barrena E, Rosset P, Muller I, Giordano R, Bunu C, et al. (2011) Bone regeneration: Stem cell therapies and clinical studies in orthopaedics and traumatology. J Cell Mol Med 15: 1266-1286.

14. Stembirek J, Kyllar M, Putnova I, Stehlik L, Buchtova M (2012) The pig as an experimental model for clinical craniofacial research. Lab Anim 46: 269-279.

15. Pearce AI, Richards RG, Milz S, Schneider E, Pearce SG (2007) Animal models for implant biomaterial research in bone: A review. Eur Cell Mater 13: 1-10.

16. Lopez MJ (2014) Bench to bedside: It's all about the model. Stem Cell Res Ther 5: 11.

17. Grisendi G, Anneren C, Cafarelli L, Sternieri R, Veronesi $\mathrm{E}$, et al. (2010) GMP-manufactured density gradient media for optimized mesenchymal stromal/stem cell isolation and expansion. Cytotherapy 12: 466-477.

18. Zuk PA, Zhu M, Mizuno H, Huang J, Futrell JW, et al. (2001) Multilineage cells from human adipose tissue: Implications for cell-based therapies. Tissue Eng 7: 211-228.

19. Veronesi E, Murgia A, Caselli A, Grisendi G, Piccinno MS, et al. (2014) Transportation conditions for prompt use of ex vivo expanded and freshly harvested clinical-grade bone marrow mesenchymal stromal/stem cells for bone regeneration. Tissue Eng Part C Methods 20: 239-251.

20. Noort WA, Oerlemans MI, Rozemuller H, Feyen D, Jaksani $S$, et al. (2012) Human versus porcine mesenchymal stromal cells: Phenotype, differentiation potential, immunomodulation and cardiac improvement after transplantation. J Cell Mol Med 16: 1827-1839.

21. Esposito M, Grusovin MG, Felice P, Karatzopoulos G, Worthington HV, et al. (2009) Interventions for replacing missing teeth: Horizontal and vertical bone augmentation techniques for dental implant treatment. Cochrane Database Syst Rev.

22. Olsen ML, Aaboe M, Hjorting-Hansen E, Hansen AK (2004) Problems related to an intraoral approach for experimental surgery on minipigs. Clin Oral Implants Res 15: 333-338.

23. Urban IA, Nagursky H, Lozada JL (2011) Horizontal ridge augmentation with a resorbable membrane and particulated autogenous bone with or without anorganic bovine bone-derived mineral: A prospective case series in 22 patients. Int $\mathrm{J}$ Oral Maxillofac Implants 26: 404-414. 